Conjugate connections and differential equations on infinite dimensional manifolds

Aghasi, M. and Dodson, C.T.J. and Galanis, G.N. and Suri, A.

2008

MIMS EPrint: 2006.8

Manchester Institute for Mathematical Sciences

School of Mathematics

The University of Manchester

\footnotetext{
Reports available from: http://eprints.maths.manchester.ac.uk/

And by contacting: The MIMS Secretary

School of Mathematics

The University of Manchester

Manchester, M13 9PL, UK
} 


\title{
CONJUGATE CONNECTIONS AND DIFFERENTIAL EQUATIONS ON INFINITE DIMENSIONAL MANIFOLDS*
}

\author{
M. AGHASI, C.T.J. DODSON, G.N. GALANIS, AND A. SURI
}

\begin{abstract}
On a smooth manifold $M$, the vector bundle structures of the second order tangent bundle, $T^{2} M$ bijectively correspond to linear connections. In this paper we classify such structures for those Fréchet manifolds which can be considered as projective limits of Banach manifolds. We investigate also the relation between ordinary differential equations on Fréchet spaces and the linear connections on their trivial bundle; the methodology extends to solve differential equations on those Fréchet manifolds which are obtained as projective limits of Banach manifolds. Such equations arise in theoretical physics. We indicate an extension of the Earle and Eells foliation theorem to the Fréchet case.
\end{abstract}

\section{INTRODUCTION}

For a smooth finite dimensional manifold $M$ the structure of $T^{2} M$, the bundle of accelerations, was studied by Dodson and Radivoiovici [4. They proved that $T^{2} M$ admits a vector bundle structure over $M$ if and only if $M$ is endowed with a linear connection. In 2] Dodson and Galanis have established the structure of $T^{2} M$ for Banach manifolds and also for those Fréchet manifolds which are projective limits of Banach manifolds. They proved that existence of a vector bundle structure on $T^{2} M$ is equivalent to the existence on $M$ of a linear connection in the sense of Vilms [16]. By this means, vector bundle structures of $T^{2} M$ were classified by Dodson, Galanis and Vassiliou for the Banach case (see [3]).

In this paper we extend that classification to a large class of Fréchet manifolds. Also, we investigate some relations between connections and ordinary differential equations on Fréchet spaces which generalize a result of Vassiliou [15] in the Banach case. As Galanis and Vassiliou have pointed out in [8], there is no specific method to solve a given differential equation on Fréchet spaces. Here we introduce a method for solving such problems and we give also a relation between these equations and the induced connections. This method can solve a wide class of ordinary differential equation on any Fréchet space because every Fréchet space can be considered as a projective limit of Banach spaces. Furthermore it extends to solve differential equations on those Fréchet manifolds which are obtained as projective limits of Banach manifolds. We indicate how the methodology may be applied by suggesting an approach to generalize the Earle and Eells [5] foliation theorem to a large class of Fréchet manifolds.

There has been recent interest in the Fréchet case for various models in theoretical physics and stochastic calculus; we mention some examples. Blair [1] studied the space $\mathcal{M}$ of all $C^{\infty}$-Riemannian metrics on a manifold $M$ as an infinite-dimensional Fréchet manifold with $C^{\infty}$-topology and provided certain of its geometrical properties. Sergeev [14 suggested a new realization of the homogeneous factor-space $\operatorname{Diff}\left(S^{1}\right) / S^{1}$, which is a Fréchet manifold. He interpreted it as the space of those

2000 Mathematical Subject Classification. Primary 58B25; Secondary 58A05

*Presented at the VIII International Colloquium on Differential Geometry, Santiago de Compostela, Spain, 7-11 July 2008. 
complex structures on the loop space $\Omega G$ of a compact Lie group $G$ (regarded as a Fréchet-Kähler manifold equipped with a canonical action of the group $\operatorname{Diff}\left(S^{1}\right)$ by symplectomorphisms) that are compatible with the symplectic structure. Minic and Tze 13 . proposed a generalization of quantum mechanics in which the projective Hilbert space of quantum events is replaced by a 'nonlinear Grassmannian' $\operatorname{Gr}\left(\mathbb{C}^{n+1}\right)$ of codimension-2 compact submanifolds of $\mathbb{C}^{n+1}$, which is a Fréchet manifold. Then it admits a symplectic structure (being a coadjoint orbit of the group of volume-preserving diffeomorphisms of $\mathbb{C}^{n+1}$ ) and a (non-integrable) almost complex structure, which make it into an almost Kähler manifold. Kinateder and McDonald 9] discussed the stochastic flow of certain diffeomorphisms via the Fréchet manifold $\mathcal{D}$ of smoothly bounded domains in $\mathbb{R}^{n}$ with compact closure; they included also a review of the relevant Fréchet geometry, developed the stochastic analysis and gave a number of examples and applications. See also McDonald [12] who studied Brownian motion in a complete Riemannian manifold $M$ where, for each $v>0$, $\mathcal{M}_{v}$ is the Fréchet manifold of all relatively compact smooth domains $D$ in $M$ of volume $v$; he obtained various results when $M$ has constant curvature.

\section{Preliminaries}

Let $M$ be a smooth manifold modelled on the Banach space $\mathbb{E}$ with the corresponding atlas $\left\{\left(U_{\alpha}, \psi_{\alpha}\right)\right\}_{\alpha \in I}$. For each $x \in M$ we define $C_{x}=\{f:(-\epsilon, \epsilon) \longrightarrow$ $M$; $\mathrm{f}$ is smooth and $\mathrm{f}(0)=\mathrm{x}\}$. For $f, g \in C_{x}$, we define $f \sim_{x} g$ iff $f^{\prime}(0)=g^{\prime}(0)$, so $T_{x} M=C_{x} / \sim_{x}$ and $T M=\bigcup_{x \in M} T_{x} M$. It is easy to check that $T M$ is a smooth Banach manifold modelled on $\mathbb{E} \times \mathbb{E}$. Moreover it is a vector bundle over $M$ by the projection $\pi_{M}: T M \longrightarrow M$. Consider the trivialization $\left\{\left(\pi_{M}^{-1}\left(U_{\alpha}\right), \Psi_{\alpha}\right)\right\}_{\alpha \in I}$ for $T M$ and similarly the trivialization $\left\{\left(\pi_{T M}^{-1}\left(\pi_{M}^{-1}\left(U_{\alpha}\right)\right), \tilde{\Psi}_{\alpha}\right)\right\}_{\alpha \in I}$ for $\mathrm{T}(\mathrm{TM})$.

Following e.g. Vilms [16, a connection on $M$ is a vector bundle morphism $\nabla: T(T M) \longrightarrow T M$ with the local forms $\omega_{\alpha}: \psi_{\alpha}\left(U_{\alpha}\right) \times \mathbb{E} \longrightarrow L(\mathbb{E}, \mathbb{E})$. Local representation of $\nabla$ is as follows:

$$
\nabla_{\alpha}: \psi_{\alpha}\left(U_{\alpha}\right) \times \mathbb{E} \times \mathbb{E} \times \mathbb{E} \longrightarrow \psi_{\alpha}\left(U_{\alpha}\right) \times \mathbb{E}
$$

with $\nabla_{\alpha}=\Psi_{\alpha} o \nabla o \tilde{\Psi}_{\alpha}^{-1}$ for $\alpha \in I$, and the relation

$$
\nabla_{\alpha}(y, u, v, w)=\left(y, w+\omega_{\alpha}(y, u) \cdot v\right)
$$

is satisfied. Furthermore $\nabla$ is a linear connection iff $\left\{\omega_{\alpha}\right\}_{\alpha \in I}$ are linear with respect to their second variables. This connection $\nabla$ is completely determined by its Christoffel symbols:

$$
\Gamma_{\alpha}: \psi_{\alpha}\left(U_{\alpha}\right) \longrightarrow L(\mathbb{E}, L(\mathbb{E}, \mathbb{E})) \equiv L_{s}^{2}(\mathbb{E} \times \mathbb{E}, \mathbb{E}) ; \alpha \in I
$$

defined by $\Gamma_{\alpha}(y)[u]=\omega_{\alpha}(y, u)$ for each $(y, u) \in \psi_{\alpha}\left(U_{\alpha}\right) \times \mathbb{E}$.

The necessary condition for $\nabla$ to be well defined on chart overlaps of $M$ is that the Christoffel symbols satisfy the following compatibility condition;

$$
\begin{aligned}
\Gamma_{\alpha}\left(\sigma_{\alpha \beta}(y)\right)\left(d \sigma_{\alpha \beta}(y)(u), d \sigma_{\alpha \beta}(y)(v)\right) & +\left(d^{2} \sigma_{\alpha \beta}(y)(v)\right)(u) \\
& =d \sigma_{\alpha \beta}(y)\left(\Gamma_{\beta}(y)(u, v)\right)
\end{aligned}
$$

for all $(y, u, v) \in \psi_{\alpha}\left(U_{\alpha}\right) \times \mathbb{E} \times \mathbb{E}$. Here $\sigma_{\alpha \beta}=\psi_{\alpha} o \psi_{\beta}^{-1}$, and $d, d^{2}$ denote the first and the second order differentials respectively.

Recalling our above definition of $C_{x}$, we define the equivalence relation $\approx_{x}$ as follows, for $f, g \in C_{x}$,

$$
f \approx_{x} g \Longleftrightarrow f^{\prime}(0)=g^{\prime}(0) \text { and } f^{\prime \prime}(0)=g^{\prime \prime}(0) .
$$


Then $T_{x}^{2} M=C_{x} / \approx_{x}$ and $T^{2} M=\bigcup_{x \in M} T_{x}^{2} M$. Here we see that $T_{x}^{2} M$ is a topological vector space isomorphic to $\mathbb{E} \times \mathbb{E}$ under the isomorphism:

$$
\begin{aligned}
\phi_{x}: T_{x}^{2} M & \longrightarrow \mathbb{E} \times \mathbb{E} \\
{[f, x]_{2} } & \longmapsto\left(\left(\psi_{\alpha} \circ f\right)^{\prime}(0),\left(\psi_{\alpha} \circ f\right)^{\prime \prime}(0)\right) .
\end{aligned}
$$

However, this identification cannot be extended to a vector bundle structure on $T^{2} M$. This can be achieved by the use of a linear connection $\nabla$ of $M$ by means of the following local trivializations:

$$
\begin{aligned}
& \Phi_{\alpha}: \pi_{2}^{-1}\left(U_{\alpha}\right) \longrightarrow U_{\alpha} \times \mathbb{E} \times \mathbb{E} \\
& {[f, x]_{2} \longmapsto\left(x,\left(\psi_{\alpha} \circ f\right)^{\prime}(0),\left(\psi_{\alpha} \circ f\right)^{\prime \prime}(0)+\Gamma_{\alpha}\left(\psi_{\alpha}(x)\right)\left(\left(\psi_{\alpha} \circ f\right)^{\prime}(0),\right.\right.} \\
& \left.\left.\left(\psi_{\alpha} \circ f\right)^{\prime}(0)\right)\right) \text {, }
\end{aligned}
$$

where $\pi_{2}: T^{2} M \longrightarrow M$ sending $[f, x]_{2}$ to $x$. In this way we see that $T^{2} M$ becomes a vector bundle over $M$ with fibres of type $\mathbb{E} \times \mathbb{E}$ and the structure group $G L(\mathbb{E} \times \mathbb{E})$.

Let $\Phi_{\alpha, x}$ be the restriction of $\Phi_{\alpha}$ to the fibres $T_{x}^{2} M$. Then the transition functions of $T^{2} M$ will be:

$$
\begin{aligned}
T_{\alpha \beta}: U_{\alpha} \cap U_{\beta} & \longrightarrow L(\mathbb{E} \times \mathbb{E}, \mathbb{E} \times \mathbb{E}) \\
x & \longmapsto \Phi_{\alpha, x} \circ \Phi_{\beta, x}{ }^{-1}
\end{aligned}
$$

More precisely, they have the form $T_{\alpha \beta}=\left(d\left(\sigma_{\alpha \beta} \circ \phi_{\beta}\right), d\left(\sigma_{\alpha \beta} \circ \phi_{\beta}\right)\right)$, for more details see [2].

\section{Classification for vector Bundle structures of $T^{2} M$}

Here we turn to a class of Fréchet manifolds that are obtained as projective limits of Banach manifolds. Let $\left\{M^{i}, \varphi^{j i}\right\}_{i, j \in \mathbb{N}}$ be a projective system of Banach manifolds modelled on the Banach spaces $\left\{\mathbb{E}^{i}\right\}_{i \in \mathbb{N}}$ respectively; we require the model spaces also to form a projective system. Suppose that for $x=\left(x^{i}\right) \in M=\lim M^{i}$ there exists a projective system of charts $\left\{\left(U_{\alpha}^{i}, \psi_{\alpha}^{i}\right)\right\}_{i \in \mathbb{N}}$ such that $x^{i} \in U_{\alpha}^{i}$ and the limit $\lim _{U_{\alpha}^{i}}^{i}$ is open in $M$. Then, the projective limit $M=\lim _{\longleftarrow} M_{i}$ has a Fréchet manifold structure modelled on $\mathbb{F}=\lim E_{i}$ with the atlas $\mathcal{A}=\left\{\left(\lim _{U_{\alpha}^{i}}^{i}, \varliminf^{\lim } \psi_{\alpha}^{i}\right)\right\}_{\alpha \in I}$. Let $\left\{M^{i}, \phi^{j i}\right\}_{i, j \in \mathbb{N}}$ and $\left\{N^{i}, \phi^{\prime j i}\right\}_{i, j \in \mathbb{N}}$ be two projective systems of manifolds, with smooth maps $g^{i}: M^{i} \longrightarrow N^{i}$ such that $\lim g^{i}=g$ exists. Suppose that for each $i \in \mathbb{N} ; M^{i}$ and $N^{i}$ are endowed with linear connections $\nabla_{M^{i}}$ and $\nabla_{N^{i}}$ which form the projective limits $\nabla_{M}=\lim _{\longleftarrow} \nabla_{M^{i}}$ and $\nabla_{N}=\lim _{\longleftarrow} \nabla_{N^{i}}$. The latter are then linear connections over $M$ and $N$ respectively.

Moreover, the next result holds:

Proposition 2.1. Let $\nabla_{M^{i}}$ and $\nabla_{N^{i}}$ be $g^{i}$-conjugate for each $i \in \mathbb{N}$ then $\nabla_{M}$ and $\nabla_{N}$ are g-conjugate.

Proof. We have to show that

$$
\nabla_{N} o T(T g)=T g o \nabla_{M}
$$

First we prove that $\lim _{N_{N^{i}} o T}\left(T g^{i}\right)$ exists.

Let $i \leq j$, then:

$$
\begin{aligned}
T \phi^{\prime j i}\left(\nabla_{N^{j}} \circ T\left(T g^{j}\right)\right) & =\left(\nabla_{N^{i}} \circ T\left(T \phi^{\prime j i}\right)\right) o\left(T\left(T g^{i}\right)\right) \\
& =\nabla_{N^{i}} \circ\left[\left(T\left(T g^{i}\right)\right) \circ T\left(T \phi^{j i}\right)\right] \\
& =\left(\nabla_{N^{i}} \circ T\left(T g^{i}\right)\right) \circ T\left(T \phi^{j i}\right),
\end{aligned}
$$

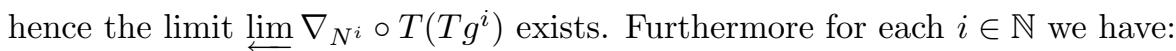

$$
T \phi^{i} \circ\left(\nabla_{N} \circ T(T g)\right)=\left(\nabla_{N^{i}} \circ T\left(T \phi^{i}\right)\right) \circ T(T g)=\nabla_{N^{i}} \circ T\left(T g^{i}\right)
$$


where $\phi^{i}: N \longrightarrow N^{i}$ are the canonical projections. As a result,

$$
\nabla_{N} \circ T(T g)=\lim _{\longleftarrow} \nabla_{N^{i}} \circ T\left(T g^{i}\right) .
$$

On the other hand, $\left\{T g^{i} \circ \nabla_{M^{i}}\right\}_{i \in \mathbb{N}}$ is a projective system of maps. Indeed for $i \leq j$ :

$$
\begin{aligned}
T \phi^{\prime j i} \circ\left(T g^{j} \circ \nabla_{M^{j}}\right) & =\left(T g^{i} \circ T \phi^{j i}\right) \circ \nabla_{M^{j}} \\
& =T g^{i} \circ\left(\nabla_{M^{i}} \circ T\left(T \phi^{j i}\right)\right) \\
& =\left(T g^{i} \circ \nabla_{M_{i}}\right) \circ T\left(T \phi^{j i}\right) .
\end{aligned}
$$

Hence $\lim ^{\log } T g^{i} \circ \nabla_{M^{i}}$ exists. Moreover:

$$
T \phi^{i} \circ\left(T g \circ \nabla_{M}\right)=\left(T g^{i} \circ T \phi^{i}\right) \circ \nabla_{M}=T g^{i} \circ \nabla_{M^{i}}, i \in \mathbb{N},
$$

where $\phi^{i}: M \longrightarrow M^{i}$ are the canonical projections of $M$. Hence

$$
T g \circ \nabla_{M}=\lim _{\longleftarrow}\left(T g^{i} \circ \nabla_{M^{i}}\right) .
$$

Based on the fact that each pair $\left(\nabla_{M^{i}}, \nabla_{N^{i}}\right), i \in \mathbb{N}$, consists of $g^{i}$-conjugate connections, we conclude that

$$
\nabla_{N} \circ T(T g)=\lim _{\longleftarrow}\left(\nabla_{N^{i}} \circ T\left(T g^{i}\right)\right)=\varliminf_{\longleftarrow}\left(T g^{i} \circ \nabla_{M^{i}}\right)=T g \circ \nabla_{M},
$$

hence $\nabla_{M}$ and $\nabla_{N}$ are indeed $g$-conjugate.

Lemma 2.2. If $\nabla_{M}=\lim _{M^{i}}, \nabla_{N}=\lim _{N_{N^{i}}}$ and $\nabla_{M^{i}}$ and $\nabla_{N^{i}}$ are $g^{i}$ conjugate, then $T^{2} g: T^{2} M \longrightarrow T^{2} N$ is linear on the fibres.

Proof. According to [3] since $\nabla_{M^{i}}$ and $\nabla_{N^{i}}$ are $g^{i}$-conjugate then $T_{x^{i}}^{2} g^{i}$ is linear for each $x^{i} \in M^{i}$. Since $T_{x}^{2} g=\varliminf_{\varliminf} T_{x^{i}}^{2} g^{i}$ the result follows.

Proposition 2.3. Let $g^{i}: M^{i} \longrightarrow N^{i}$ be smooth maps and $\nabla_{M^{i}}$ and $\nabla_{N^{i}}$ be $g^{i}$ conjugate for each $i \in \mathbb{N}$. Then, $T^{2} g: T^{2} M \longrightarrow T^{2} N$ is a vector bundle morphism.

Sketch of proof. As proved in [3], each $T^{2} g^{i}: T^{2} M^{i} \longrightarrow T^{2} N^{i}$ is a vector bundle morphism for each $i \in \mathbb{N}$; and since $T^{2} g=\lim T^{2} g^{i}$, we get the result.

In view of the above discussion, we deduce the following main result:

Theorem 2.4. Let $g^{i}: M^{i} \longrightarrow M^{i}$ be a diffeomorphism and $\nabla^{i}$ and $\nabla^{\prime i} g^{i}$ conjugate linear connections on $M^{i}$, for each $i \in \mathbb{N}$. If $\nabla=\varliminf_{\lim } \nabla^{i}$ and $\nabla^{\prime}=\varliminf_{\text {lim }} \nabla^{\prime i}$, then the vector bundle structures on $T^{2} M$ induced by $\nabla$ and $\nabla^{\prime}$ are isomorphic.

Let $(M, \nabla)$ denote the vector bundle structure of $T^{2} M$ induced by $\nabla$. For a diffeomorphism $g: M \longrightarrow M$ we define the equivalence relation $\sim_{g}$ as follows:

$$
(M, \nabla) \sim_{g}\left(M, \nabla^{\prime}\right) \Longleftrightarrow \nabla \text { and } \nabla^{\prime} \text { are } g \text { - conjugate. }
$$

Hence if $(M, \nabla)$ and $\left(M, \nabla^{\prime}\right)$ are in the same g-conjugate class $[(M, \nabla)]_{g}$, their induced vector bundle structures on $T^{2} M$ are isomorphic.

Corollary 2.5. All the elements of the class $[(M, \nabla)]_{g}$ have isomorphic induced vector bundle structures on $T^{2} M$.

\section{Connections And Ordinary Differential Equations}

Let $\mathbb{E}$ and $\mathbb{B}$ be Banach spaces and $L=\left(\mathbb{E} \times \mathbb{R}, \mathbb{R}, p r_{1}\right)$ be the trivial bundle over $\mathbb{R}$ with fibres of type $\mathbb{E}$. In [15] it is stated that we can correspond an ordinary differential equation to a connection over the trivial bundle with the solution $\xi$ being the horizontal global section of the obtained connection. Furthermore, it is shown that connections $\nabla$ and $\nabla^{\prime}$ over $L$ are conjugate iff the corresponding differential equations $d x / d t=A(t) x$ and $d x / d t=B(t) y$ are equivalent. 
Here we extend these concepts to Fréchet spaces. Let $\mathbb{F}$ be a Fréchet space with $\mathbb{F}=\lim _{\longleftarrow}\left\{\mathbb{E}^{i}, \rho^{j i}\right\}_{i, j \in \mathbb{N}}$. Consider the trivial bundle $L=\left(\mathbb{R} \times \mathbb{F}, \mathbb{R}, p r_{1}\right)$ with respect to the usual atlas $\mathcal{A}$ for $\mathbb{R}$ formed by the global chart $\left(\mathbb{R}, i d_{\mathbb{R}}\right)$.

Assume that $\nabla^{i}$ is a linear connection over $L^{i}=\left(\mathbb{R} \times \mathbb{E}^{i}, \mathbb{R}, p r_{1}\right)$ and that the corresponding Christoffel symbols commute with the connecting morphisms $\rho^{j i}$. Then, $\nabla=\lim \nabla^{i}$ a linear connection on $L=\lim _{\longleftarrow} L^{i}$ characterized by a single Christoffel symbol:

$$
\Gamma: \mathbb{R} \longrightarrow L^{2}(\mathbb{R} \times \mathbb{F}, \mathbb{F}) .
$$

Let $A(t)=\Gamma(t)(., 1)$ where 1 is the unit of $\mathbb{R}$. Then the following result holds true:

Theorem 3.1. Linear connections of the above type are in one-to-one correspondence with the ordinary differential equations $d x / d t=A(t) x$ where the factor $A$ is obtained as a projective limit. Moreover for each $t_{0} \in \mathbb{R}$ there exists a unique horizontal global section

$$
\xi: \mathbb{R} \longrightarrow \mathbb{R} \times \mathbb{F}
$$

with $\xi_{p}\left(t_{0}\right)=f_{0}$, where $\xi_{p}: \mathbb{R} \longrightarrow \mathbb{F}$ is the principal part of $\xi$.

Proof. We know that $\nabla=\lim \nabla^{i}$ such that each $\nabla^{i}$ is a linear connection over $L^{i}$. As it is stated in [15] each $\nabla^{i}$ corresponds bijectively to an ordinary differential equation $d x^{i} / d t=A^{i}(t) x^{i}$. Furthermore, any solution of $d x^{i} / d t=A^{i}(t) x^{i}$ is the principal part of the horizontal global section of $\nabla^{i}$, which we call $\xi^{i}$.

We notice firstly that $A^{i}\left(t^{i}\right)=\Gamma^{i}\left(t^{i}\right)(., 1)$ where $\Gamma^{i}$ is the Christoffel symbol $\nabla^{i}$ over $L^{i}$ assigned to the chart $\left(\mathbb{E}^{i}, i d_{\mathbb{E}^{i}}\right)$. Since $\nabla=\lim ^{i} \nabla^{i}$ we get $\Gamma(t)(., 1)=$

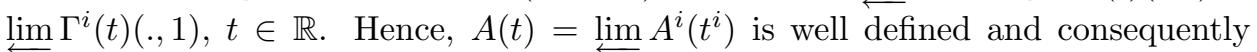
$\overleftarrow{d x} / d t=A(t) x$ is an ordinary differential equation on $\mathbb{E}^{i}$. (For more details see [6].)

Let $\xi_{p}^{i}$ be the solution of $d x / d t=A_{i}(t) x$, satisfying $\xi_{p}^{i}\left(t_{0}\right)=f_{0}^{i}$. We claim that $\left\{\xi_{p}^{i}\right\}_{i \in \mathbb{N}}$ is a projective system of maps and $\xi_{p}=\lim _{\longleftarrow} \xi_{p}^{i}$ is the solution of $d x / d t=A(t) x$. One has first to check that:

$$
\rho^{j i} o \xi_{p}^{j}=\xi_{p}^{i}
$$

for $i \leq j$. To this end, we see that

$$
\begin{aligned}
\left(\rho^{j i} \circ \xi_{p}^{j}\right)^{\prime}(t) & =\rho^{j i} \circ\left(\xi_{p}^{j}\right)^{\prime}(t)=\rho^{j i} \circ\left[A^{j}(t)\right]\left(\xi_{p}^{j}(t)\right) \\
& =\left[\rho^{j i} \circ A^{j}(t)\right]\left(\xi_{p}^{j}(t)\right) \\
& =\left[A^{i}(t) \circ \rho^{j i}\right]\left(\xi_{p}^{j}(t)\right) \\
& =\left[A^{i}(t)\right]\left(\xi_{p}^{i}(t)\right)
\end{aligned}
$$

Moreover, $\xi_{p}^{i}\left(t_{0}^{i}\right)=f_{0}^{i}$ and $\left(\rho^{j i} o \xi_{p}^{j}\right)\left(t_{0}\right)=\rho^{j i}\left(f_{0}^{j}\right)=f_{0}^{i}$. Based on the uniqueness of the solutions of differential equations on Banach spaces over given initial conditions, we conclude that $\rho^{j i} \circ \xi_{p}^{j}=\xi_{p}^{i}$. This implies that $\xi_{p}=\lim _{\longleftarrow} \xi_{p}^{i}$ exists. Furthermore, it is the solution of the above-mentioned differential equation:

$$
\begin{aligned}
\xi_{p}^{\prime}(t)=\left(\xi_{p}^{i^{\prime}}\left(t^{i}\right)\right)_{i \in \mathbb{N}} & =\left(A^{i}\left(t^{i}\right)\left(\xi_{p}^{i^{\prime}}\left(t^{i}\right)\right)\right)_{i \in \mathbb{N}} \\
& =A(t)\left(\xi_{p}(t)\right) .
\end{aligned}
$$

Similar calculations ensure that $\xi_{p}=\lim \xi_{p}^{i}$ is also the unique horizontal global section of $\nabla$ as a projective limit of global sections.

Let $\nabla=\lim _{\longleftarrow} \nabla^{i}$ and $\nabla^{\prime}=\lim ^{\prime i} \nabla^{\prime i}$ be two linear connections over $L$ such that for

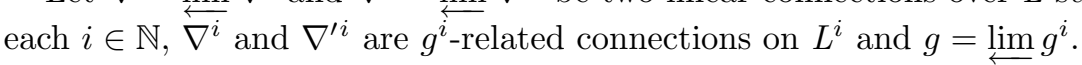


Theorem 3.2. With the same assumptions, let $\nabla=\lim \nabla^{i}$ and $\nabla^{\prime}=\lim \nabla^{\prime i}$ be two linear connections over $L$. Then $\nabla$ and $\nabla^{\prime}$ are $\left(g, i d_{\mathbb{R}}\right)$-related iff their corresponding differential equations, given by $d x / d t=A(t) x$ and $d y / d t=C(t) y$, are equivalent i.e. there exists a smooth transformation $Q: \mathbb{B} \longrightarrow \mathcal{H}^{0}(\mathbb{F})$ such that $x(t)=Q(t) y(t)$ or equivalently,

$$
C(t)=Q^{-1}(t) \circ(A(t) \circ Q(t)-\dot{Q}(t))
$$

for each $t \in \mathbb{R}$.

Proof. By [15] $\nabla^{i}$ and $\nabla^{\prime i}$ are $g^{i}$-related connections over $L^{i}$ iff $d x^{i} / d t=A^{i}(t) x^{i}$ and $d y^{i} / d t=C^{i}(t) y^{i}$ are equivalent i.e.

$$
C^{i}(t)=\left(Q^{i}\right)^{-1}(t) \circ\left(A^{i}(t) \circ Q^{i}(t)-\dot{Q}^{i}(t)\right): i \in \mathbb{N},
$$

where $Q=\epsilon \circ Q^{*}, Q^{*}=\left(Q^{i}\right)_{i \in \mathbb{N}}$ and $\epsilon$ is the natural morphism

$$
\begin{aligned}
& \epsilon: \mathcal{H}^{0}(\mathbb{F}) \longrightarrow \mathcal{L}(\mathbb{F}) \\
&\left(l^{i}\right)_{i \in \mathbb{N}} \longmapsto \\
& \lim l^{i}
\end{aligned}
$$

Hence (1) implies that: $\nabla^{i}$ and $\nabla^{\prime i}$ are $g^{i}$-related iff $x(t)=Q(t) y(t)$.

Note that the existence of intrinsic obstacles in the structure of the space of continuous linear mappings $\mathcal{L}(\mathbb{F})$, which drops out of the category of Fréchet spaces, leads us to replace it with the Fréchet space $\mathcal{H}(\mathbb{F})$.

$$
\mathcal{H}(\mathbb{F})=\left\{\left(l^{i}\right)_{i \in \mathbb{N}} \in \prod_{i=1}^{\infty} \mathcal{L}\left(\mathbb{E}^{i}\right): \lim _{\longleftarrow} l^{i} \text { exists }\right\}
$$

More precisely, $\mathcal{H}(\mathbb{F})$ can be considered as the projective limit of the Banach spaces:

$$
\mathcal{H}_{i}(\mathbb{F}):=\left\{\left(l^{1}, \ldots, l^{i}\right) \in \prod_{j=1}^{i} \mathcal{L}\left(\mathbb{E}^{j}\right): \rho^{j k} \circ l^{j}=l^{k} \circ \rho^{j k} ; \text { for } k \leq j \leq i\right\} .
$$

The work presented in this paper can be applied in order to obtain a potentially useful Floquet - Liapunov theorem in Fréchet spaces:

Corollary 3.3. Let $\nabla=\lim _{\longleftarrow} \nabla^{i}$ be a linear connection over $L$ with periodic coefficient $A$. Then there exist $\overleftarrow{a}$ linear connection $\nabla^{\prime}$ with constant Christoffel symbols, where $\nabla$ and $\nabla^{\prime}$ are $\left(g, i d_{\mathbb{R}}\right)$ - related.

Proof. According to [8] the differential equation $\dot{x}(t)=A(t) x(t)$ with periodic coefficient $\mathrm{A}$ is equivalent with the differential equation $\dot{y}(t)=B(t) y(t)$ such that $\mathrm{B}$ is constant. Let $\nabla^{\prime}$ be the linear connection over $\mathrm{L}$ assigned to $\mathrm{B}$ then, by Theorem $3.2, \nabla$ and $\nabla^{\prime}$ are $\left(g, i d_{\mathbb{R}}\right)-$ related.

\section{The Earle and Eells Foliation Theorem in Fréchet spaces}

The target here is to indicate a possible generalization of the following result to a wide class of Fréchet manifolds:

Theorem 4.1 (Earle and Eells [5]). Let $(X, \alpha),(Y, \beta)$ be Finsler $C^{1}$-manifolds modeled on Banach spaces, and suppose that $(X, \alpha)$ is complete. Let $f: X \rightarrow Y$ be a surjective $C^{1}$-map which foliates $X$. If there is a, locally bounded over $Y$, Lipschitz splitting of the sequence

$$
0 \longrightarrow \operatorname{Ker} f_{*} \longrightarrow T X \stackrel{f_{*}}{\longrightarrow} f^{-1}(T Y) \longrightarrow 0,
$$

where $f_{*}$ stands for the differential of $f$ at $x$, then $f$ is a locally $C^{0}$-trivial fibration. 
The proof of Theorem 4.1 is strongly based on properties of differential equations in Banach spaces, used to construct coherent liftings of paths. As a result, any attempt to generalize it to the Fréchet framework encounters serious difficulties. This is because the local structure of the space models do not admit a general solvability theory for ordinary differential equations analogous to that of the Banach case. Indeed, in a Fréchet space an initial value problem may have no solution, a single one or multiple solutions.

A way out of these difficulties is proposed here for a wide class of Fréchet manifolds: those that can be obtained as projective limits of Banach corresponding factors (see [7, 2]). To be more precise, we consider the manifolds $X, Y$ to be limits of a projective system of Banach Finsler manifolds: $X=\lim \left\{\left(X^{i}, \alpha^{i}\right)\right\}_{i \in \mathbb{N}}$, $Y=\lim \left\{\left(Y^{i}, \beta^{i}\right)\right\}_{i \in \mathbb{N}}$. Then, suppose that $X$ and $Y$ can be endowed with general-

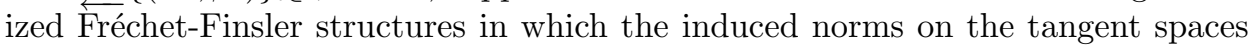
are replaced by sequences of semi-norms.

If this is the case, then a corresponding limit of mappings $f=\lim f^{i}: X \rightarrow Y$ satisfying the properties of the previous theorem, can be realized $\longleftarrow$ as a projective limit of $C^{1}$-factors $f^{i}: X^{i} \rightarrow Y^{i}, i \in \mathbb{N}$, where each of factor satisfies also the assumptions of Theorem 4.1. It follows that a sequence of $C^{0}$-trivial fibrations will be obtained and it will projectively converge to $f$. Taking into account that the notion of triviality on the fibers is compatible with projective limits, one obtains this property also for $f$, avoiding the use of the pathological differential equations on Fréchet spaces.

Note that if the domain manifold $X$ is assumed to be Banach modeled and $Y$ a Finsler manifold as above, then the result obtains for every surjective $C^{1}$-map $f$ which foliates $X$.

\section{REFERENCES}

[1] D.E. Blair, Spaces of metrics and curvature functionals, in Handbook of differential geometry, Vol. I, 153-185, North-Holland, Amsterdam, 2000.

[2] C.T.J. Dodson and G.N. Galanis, Second order tangent bundles of infinite dimensional manifolds, J. Geom. Phys., 52 (2004), pp. 127-136.

[3] C.T.J. Dodson, G.N. Galanis and E. Vassiliou, Isomorphism classes for Banach vector bundle structures of second tangents, Math. Proc. Camb. Phil. Soc., 141 (2006), pp. 489-496.

[4] C.T.J. Dodson and M.S.Radivoiovici, Tangent and frame bundle of order two,, Analele stiintifice ale Universitatii "Al. I. Cuza", 28(1982),63-71.

[5] C. J. Earle and J. Eells Jr., Foliations and Fibrations, J. Differential Geometry, 1 (1967), 33-41.

[6] G.N. Galanis, Differential and Geometric Structure for the Tangent Bundle of a Projective Limit Manifold, Rendiconti del Seminario Matematico di Padova, Vol. 112 (2004).

[7] G. Galanis, Projective Limits of vector bundles, Portugaliae Mathematica 55 (1998), 11-24.

[8] G.N. Galanis and E. Vassiliou, A Floquet-Liapunov theorem in Fréchet spaces, Annali della Scuola Normale Superiore di Pisa (4), 27 (1998), 427-436.

[9] K. Kinateder and P. McDonald, An Ito formula for domain-valued processes driven by stochastic flows. Probability Theory and Related Fields 124 1, (2002), 73-99.

[10] S. Lang, Differential manifolds, Addison-Wesley, Reading Massachusetts, 1972.

[11] J. A. Leslie, Some Frobenious theorems in Global Analysis, J. Diff. Geom. 42 (1968), 279-297.

[12] P. McDonald, Isoperimetric conditions, Poisson problems, and diffusions in Riemannian manifolds. Potential Analysis 16, 2 (2002), 115-138

[13] D. Minic and C-H. Tze, A general theory of quantum relativity. Phys. Lett. B 581 1-2, (2004) 111-118.

[14] A. Sergeev, Diff $+\left(S^{1}\right) / S^{1}$ as a space of complex structures on loop spaces of compact Lie groups. Stochastic processes, physics and geometry: new interplays, II (Leipzig, 1999), 573588, CMS Conf. Proc., 29, Amer. Math. Soc., Providence, RI, 2000.

[15] E. Vassiliou, Transformations of Linear Connections, Period. Math. Hungar. 13(4) (1982), 286-308.

[16] J. Vilms, Connections on tangent bundles, J. Diff. Geom. 41 (1967), 235-243. 
Department of Mathematics, Isfahan University of Technology, Isfahan, Iran

E-mail address: m.aghasi@cc.iut.ac.ir

School of Mathematics Manchester University, Manchester, M13 9PL, United KingDOM

E-mail address: ctdodson@manchester.ac.uk

Section of Mathematics, Naval Academy of Greece, Xatzikyriakion, Piraeus 18539 , Greece

E-mail address: ggalanis@snd.edu.gr

Department of Mathematics, Isfahan University of Technology, Isfahan, Iran

E-mail address: a.suri@math.iut.ac.ir 